\title{
Research on the Spatial Pattern of Forest Undergrowth Industries Based on the Evaluation of Forest Ecosystem Services in Langxiang Town
}

\author{
Ang $\mathrm{Li}^{1,2}$, Tianyu $\mathrm{Zhao}^{1,2^{*}}$ \\ ${ }^{1}$ School of Architecture, Department of Rural \& Urban Planning, Harbin Institute of Technology, Harbin 150006, China \\ ${ }^{2}$ Key Laboratory of Science and Technology of Urban and Rural Residential Environment in Cold Region of Ministry of \\ Industry and Information Technology, Harbin 150001, China
}

Corresponding Author Email: 15B334017@hit.edu.cn

https://doi.org/10.18280/ijdne.150315

Received: 22 January 2020

Accepted: 11 May 2020

\section{Keywords:}

forest ecosystem, evaluation of ecosystem services, forest undergrowth industries, spatial pattern, Langxiang town

\begin{abstract}
Taking Langxiang town as a case, this paper presents a way to coordinate the industrial and ecological space from the perspective of ecosystem services (ES). According to relevant theories and methods of ES, the forest ES are classified into 4 major functions: supply, support, regulation and aesthetic landscape service, and 14 specific indicators are included to construct an ES evaluation system applicable to forest resource-based towns in northeast China. We summarize 8 geospatial and biological factors influencing forest ES spatial heterogeneity to estimate relative ES values, and take the ES needs of different forest undergrowth industries (FUI) as the weights. The spatial suitability zoning map of each industry is obtained by operating weighted overlay analysis on GIS platform, the advantages and constraints of FUI spatial development are expressed visually. We construct the comprehensive spatial pattern of the FUI system using the maps of various industries, the results show that the south of the town has a larger development space, which is contrary to the urban spatial pattern, the multifunctional areas and industrial axes are also identified.
\end{abstract}

\section{INTRODUCTION}

In the process of China's new urbanization, the problem of transitional development of forest resource-based towns is particularly prominent. These areas not only carry important ecological functions for the sta424te, but also rely on forestry in both economic and social structure for a long time [1]. Relevant studies have shown that the "resource curse" hypothesis has become a reality in these towns [2], that is, the richer the forest resources, the lower the level of economic development. Moreover, the state policy of total ban on commercial logging accelerates the problem.

Under the dual pressure of urban development and ecological protection, the forest undergrowth industries (FUI) which take the non-wood products as the core have gradually become the pillar industries of these towns. To some extent, the new industries have solved some issues such as economic difficulties, forest workers resettlement, and forest restoration [3]. However, new problems also come out: aimless development, undiversified products, lose-lose competition between the companies, ecological damage, for example. These have greatly hindered the sound and coordinating development of FUI [4]. The basic reasons are: (1) there are lacks of understandings of the endowment characteristics and capacity of regional forest resources, (2) there is no evaluation on the ecological needs and impacts of FUI, (3) the most directly, there is no spatial pattern layout about FUI as a support.

Ecosystem service (ES) is "the benefit that human beings derive directly or indirectly from all the ecosystem functions" [5], evaluation ES is an effective mean to coordinate the relationship between human development and natural resources. In 2005, Millennium Ecosystem Assessment (MEA) proposed the basic paradigm of ES evaluation, which divides ES into four functions: supply, support, regulation and culture [6], these functions can be valued to describe the importance of different ecosystems, and it has become a widely accepted theoretical framework. Since then, many ecologists have conducted relevant research on the value of forest ES [7-9], Xie et al. estimated the value of forest ES in China to be 17.53 trillion yuan [10]. Most research on forest ES focused on the accurate calculation of value [11-13]. However, these results are still difficult to apply the actual spatial management of forestry, this is because the forest ES as a kind of ecological properties, has a strong spatial heterogeneity [14], even for two consecutive forests, their ES value are always different as influenced by many factors. Although some researchers discussed the spatial heterogeneity of ES $[15,16]$, few case combined it with local industry spatial planning on a small scale.

Besides the value of forest ES, another important factor in determining the spatial distribution of FUI is the diverse needs for ES. Due to the disparate production modes and diverse influences on forest environment, there are differences in spatial suitability of FUI, for example, there are obvious different needs for the forest culture service between tourism and underwood planting. Therefore, integrating the spatial heterogeneity of forest ES and the diverse needs of FUI is necessary for optimizing the spatial pattern.

Based on these backgrounds and existing research results, we take Langxiang town, a typical forest resource-based town in northeast China, as a case to evaluate its forest ES based on 
spatial heterogeneity, and apply the results to the construction of FUI spatial pattern for the first time, so as to achieve the balance between forest ecosystem and industrial development. The remainder of this paper is organized as follows. Section 2 introduces the overview of Langxiang town and puts forward the research methods. Section 3 presents the evaluation indicator system and the mapping process of ES value. Section 4 supplies the need degree for ES functions of various FUI. Section 5 combines the results of section 3 and section 4 to obtain the suitability zoning in forest land for each FUI, and comprehensively forms the distribution pattern guiding the spatial development of FUI in Langxiang. Section 6 gives the conclusion.

\section{RESEARCH AREA AND METHODS}

\subsection{Research area}

Langxiang town (Figure 1) is located in the southern foot of Xiao Hinggan Mountains, Heilongjiang Province, with a total area of 2,647.0 $\mathrm{Km}^{2}$. It is rich in forest resources with a forest land area of $2,538.8 \mathrm{Km}^{2}$ (nearly $95.9 \%$ of the total area). It is a typical forest resource-based town with mixed management of both forestry department and local government. The forest type belongs to the plant partitioning system of the remnant vein of Changbai Mountain. The hills and rivers are widely and complicatedly distributed, the average slope is about $12^{\circ}$ and the maximum slope is about $45^{\circ}$. Soil and vegetation are vertically differentiated, the soil is mainly to dark brown soil. The town has a typical coniferous and broad-leaved mixed forest area with temperate and subtropical vegetation types [17]. Due to long years of over-logging and the artificial replanting in recent years, the forest ecosystems have undergone major changes and the ecological matrices are uneven and intermixed now.

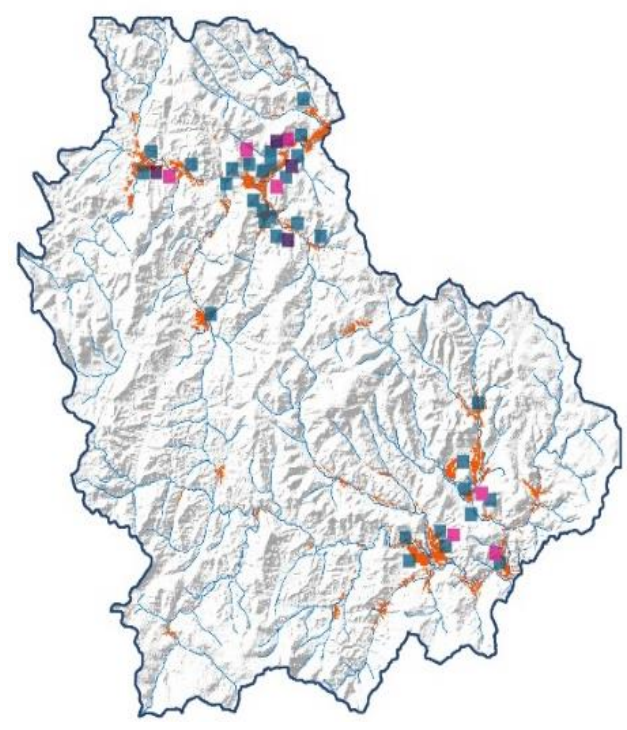

Figure 1. Overview of the lands and forest industries distribution in Langxiang Town

Although the proportion of agricultural land in the township is very low, its industrial development is still dominated by the primary industry, of which the proportion of forestry and forest husbandry output value is $53.9 \%$. In recent years, Langxiang town is committed to industrial economic transformation from the traditional resource-based town to the town led by tourism and FUI. According to the economic data [18] (Table 1, Table 2), it can be known that the town's forest economy is significant but growths slowly. As the outputs are obviously inconsistent with the forest scale, there is a big space for development. Besides, the prospect of forest tourism is better.

Table 1. Outputs of main forest products, 2015

\begin{tabular}{cccc}
\hline Forest products & Outputs & $\begin{array}{c}\text { Forest } \\
\text { products }\end{array}$ & $\begin{array}{c}\text { Outputs } \\
\text { (Pcs) }\end{array}$ \\
\hline Forest oil crops & $80 \mathrm{Tons}$ & Goats & 12,710 \\
Forest herbs & $254 \mathrm{Ha}$ & Poultries & 211,900 \\
Dried black fungus & $2,977 \mathrm{Tons}$ & Bee hives & 4,770 \\
Dried mushrooms & $1,467 \mathrm{Tons}$ & Deers & 1,353 \\
Dried antlers & $1,001 \mathrm{Kg}$ & Raccoons & 1,635 \\
\hline
\end{tabular}

Table 2. Output values of main industrial types, 2014-2015

\begin{tabular}{cccc}
\hline $\begin{array}{c}\text { Industrial } \\
\text { types }\end{array}$ & $\begin{array}{c}\mathbf{2 0 1 4} \\
\text { (million yuan) }\end{array}$ & $\begin{array}{c}\mathbf{2 0 1 5} \\
\text { (million yuan) }\end{array}$ & $\begin{array}{c}\text { Growth } \\
\text { rate }\end{array}$ \\
\hline Forestry & 204.64 & 219.92 & $6.5 \%$ \\
Livestock & 184.61 & 199.02 & $7.8 \%$ \\
Agriculture & 684.75 & 777.52 & $13.5 \%$ \\
Tourism & 114.29 & 143.00 & $25.1 \%$ \\
\hline
\end{tabular}

\subsection{Methods}

The essence of the optimization of industrial spatial pattern is the selection of the most suitable space, which is the result of the comprehensive action of a collection of related factors. We design an evaluation model to realize this process, the process flow is shown in Figure 2, and four key steps are as follow:

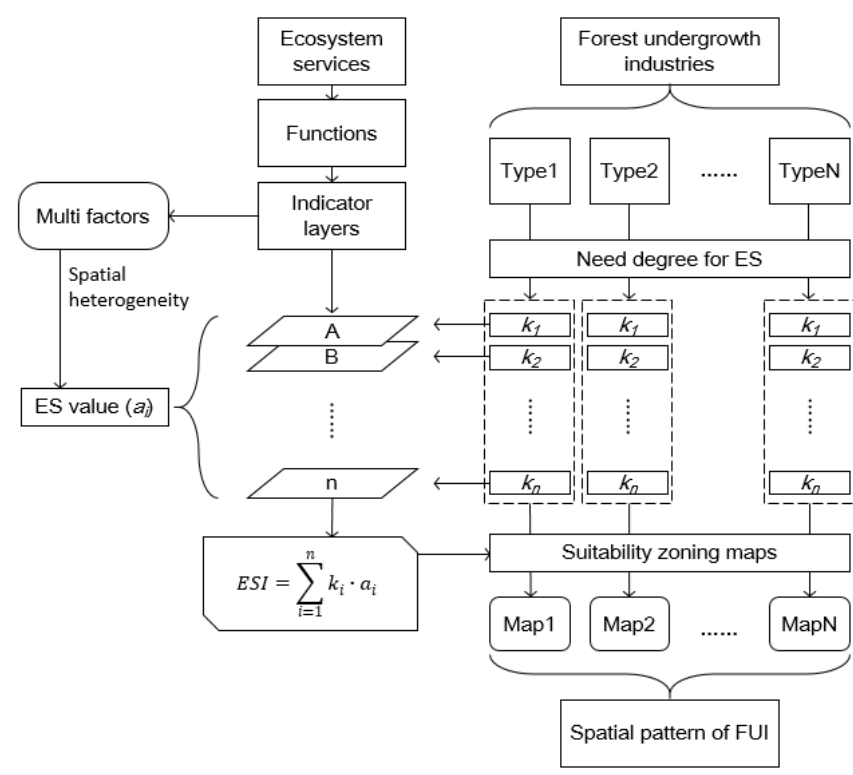

Figure 2. Process flow of the methods

(1) Classification of the ES functions. Take ecological service evaluation as the overall aim, considering the direct relevance of industrial development, divide it into four major functions: supply, support, regulation and aesthetic landscape service. Each function is subdivided into several indicators for description. The supply services include the production factors directly provided by the forest. The support services refer to 
the physical and geographical conditions. The regulation services represent the ecological restoration capabilities or ecological tolerance factors that the forest ecosystem can provide. The aesthetic landscape services are mainly aimed at the landscape resources needed for the development of the tourism industry.

(2) Identification and quantification of the factors. According to related studies [19] and the Technical Specification for Forest Resources Survey [20] of China, we identify the significant factors which cause the space heterogeneity of each indicators from step (1). The spatial data of the factors are collected from satellite images and local forestry bureau. After standardization, each factor can form a score layer by threshold dividing. The weight of each layer is calculated by AHP method. For every pixel of the town space, the relevant value of ES indicator is equal to the sum of weighted scores.

(3) Need degree for ES functions. According to the production materials and methods, combining with the current situation of the town, different types of FUI can be listed. Through experts scoring, the need degree of each industry type for different ES functions is distinguished and calculated as a weight between $0-5$.

(4) Mapping of ES index. Using the values and weights of various indicators calculated from step (2) and (3), we do the weighted overlay analysis on GIS, then the ES index map is obtained for every type of FUI. Every pixel in the map has its own ES index value, and the larger this value, the spatial suitability of the industry is greater. Mathematically, The ES index is given by:

$$
E S I=\sum_{i=1}^{n} k_{i} \cdot a_{i}
$$

where, ESI is the ES index of a FUI type, $k_{i}$ is the weight (i.e. the need degree) of ES indicator $i, a_{i}$ is the relevant value (i.e. the weighted score) of ES indicator $i, n$ is the total number of ES indicators influential to this type of FUI.

\section{EVALUATION OF ES VALUE}

\subsection{Structure and explanation of indicator system}

We change the culture service to the aesthetic landscape service because of the relevance between forest ES and FUI, and the other services are same to the classification of MEA [6]: supply, support and regulation services. The subdivision functions are mainly based on the characteristics of the forest ecosystem of Langxiang town.

Indicators of supply services include: Available woodland space (A). It indicates available space which allow the FUI to use, it includes forest-covered space or non-forest land which is suitable for afforestation. Biomass resources (B), including non-wood resources such as fruits, deciduous forests, other animal and plant resources, microorganisms, and humus that forests can provide, and their contents are mainly affected by forest biomass. Abundance of species (C). It is a potential resource that is mainly targeted at the types of industries that have special requirements for biodiversity.
Indicators of support services include four basic geographical condition indicators: slope (D), aspect (E), elevation $(\mathrm{F})$ and soil type $(\mathrm{G})$, these indicators have a direct impact to the FUI location.

The indicators of regulation services include: Soil and water conservation $(\mathrm{H})$. It has a significant meaning for the underwood agriculture, also this indicator can be used to limit the industries that are probably to cause soil erosion setting up on the forest lands with low service levels. Air purification (I). Forest land with a higher level of this indicator has higher tolerance to forest industries that generate large amounts of $\mathrm{CO}_{2}$ and other polluting gas. Degradation of pollutants $(\mathrm{J})$. It refers to the natural degradation of solid-liquid pollution, it also stands for how stable an ecosystem is. Microclimatic protection $(\mathrm{K})$. The capacity of protecting the undergrowth space against wind, rainfall, solar radiation, snowfall and other natural factors has significant impacts on certain forest industries.

The aesthetic landscape services include: summer (L), autumn $(\mathrm{M})$, and winter landscape $(\mathrm{N})$. The indicators are set based on the characteristics of the major tourism resources in Langxiang. Since the special climate that the spring is much shorter, in addition, there has no obvious seasonal characteristics and natural landscape resources in this period, therefore spring is not included in the aesthetic landscape service index. The summer landscape can provide forest river drift and virgin forest sightseeing. In autumn, the town is famous for "Colorful Mountains" as a local tradition. In winter, the mainly tourism activities are ice and snow sports. As a result, there are specific landscape service needs for different tourism activities in different seasons.

\subsection{Identification and contribution rate of factors}

The value of ES within any region, regardless of any measurement method, obviously depends on the attributes of the ecosystem, and these attributes are determined by a set of natural and geographical factors. Due to the spatial inhomogeneity of the factors, ES value presents a spatial heterogeneity. That means the ES value can be measured by quantification of the factor value and weight.

Using the method in section 2.2, 8 significant factors affecting the level of forest ES are identified: slope (I), aspect (II), elevation (III), canopy density (IV), forest origin (V), species composition (VI), soil type (VII), and water distribution (VIII). Among them, the forest canopy density refers to the ratio of the vertical projection area of the canopy to the forest land area; the forest origin describes the degree of forestry artificiality; the tree species composition reflects the proportion of the tree species within the forest class. The spatial data is obtained from The Fifth Forest Survey of China.

Each factor has impacts on related ES indicators, but the depth of the impacts depends, so we use the contribution rate to represent it. Through the questionnaire survey of 50 forest experts and forest managers, we get an average judgment matrix to calculate the eigenvector of each factor by means of AHP, and that is the contribution rate to the ES indicators of each factor (Table 3). The relevance between ES indicators, factors and major ES functions is showed as Figure 3, the results show the canopy density, forest origin and species composition play more important roles to the constitution of ES value. 
Table 3. Contribution rates of the factors for ES indicators

\begin{tabular}{lccccccccc}
\hline & I & II & III & IV & V & VI & VII & VIII & Tot \\
\hline $\mathbf{A}$ & 0.29 & 0 & 0 & $0.38^{*}$ & 0.13 & 0.10 & 0 & 0 \\
$\mathbf{B}$ & 0 & 0 & 0 & 0.31 & 0.16 & 0.28 & 0.25 & 0 \\
$\mathbf{C}$ & 0 & 0 & 0.14 & 0.29 & 0.11 & 0.33 & 0 & 0.13 \\
$\mathbf{D}$ & 1 & 0 & 0 & 0 & 0 & 0 & 0 & 0 \\
$\mathbf{E}$ & 0 & 1 & 0 & 0 & 0 & 0 & 0 & 0 \\
$\mathbf{F}$ & 0 & 0 & 1 & 0 & 0 & 0 & 0 & 0 & 1 \\
$\mathbf{G}$ & 0 & 0 & 0 & 0 & 0 & 0 & 1 & 0 & 1 \\
$\mathbf{H}$ & 0.25 & 0 & 0 & 0.53 & 0 & 0 & 0.12 & 0.10 & 1 \\
$\mathbf{I}$ & 0 & 0 & 0 & 0.66 & 0 & 0.34 & 0 & 0 & 1 \\
$\mathbf{J}$ & 0 & 0 & 0 & 0.43 & 0 & 0.20 & 0.37 & 0 & 1 \\
$\mathbf{K}$ & 0 & 0.18 & 0 & 0.69 & 0 & 0.13 & 0 & 0 \\
$\mathbf{L}$ & 0.06 & 0 & 0 & 0.06 & 0.61 & 0 & 0 & 0.27 & 1 \\
$\mathbf{M}$ & 0 & 0.15 & 0 & 0.31 & 0 & 0.54 & 0 & 0 \\
$\mathbf{N}$ & $0.40^{*}$ & 0.30 & 0 & $0.17^{*}$ & $0.13^{*}$ & 0 & 0 & 0 \\
\hline
\end{tabular}

Notes: number with * means negative correlation.

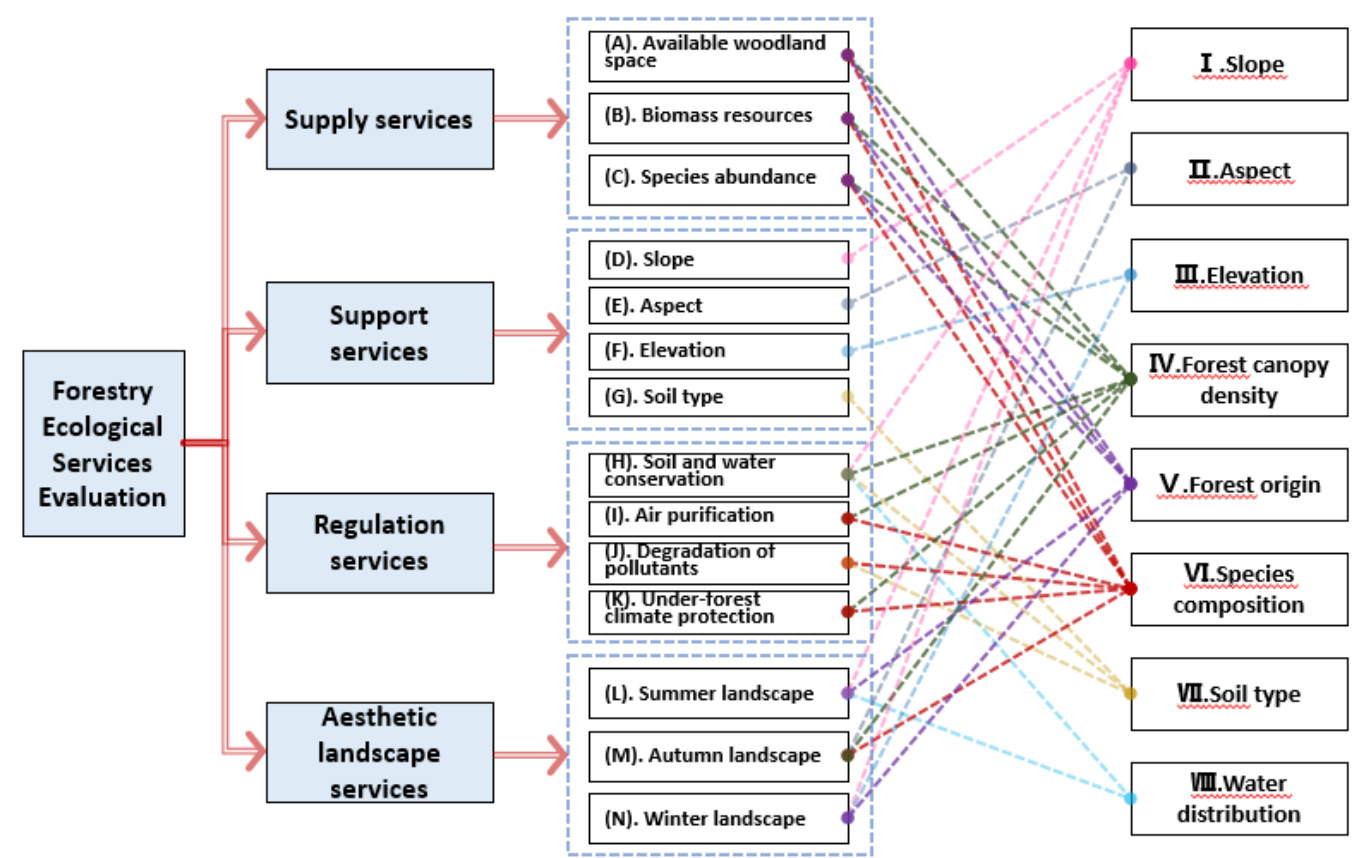

Figure 3. Relevance between the indicators and factors

\subsection{Standardization of the factors}

As the inconsistent measurement standards of each factor, it is necessary to standardize it. For factors with specific values such as slope and elevation, the threshold is divided equally. For factors without value data, they are qualitatively divided. According to related researches [21], the overall principle is that if the value change of an area leads to a stronger ES function, the higher score this area will have. However, there are exceptions, for example, the higher the forest canopy density is, the smaller available forest space there will be, in this case, the factor and indicator are negatively correlated. Accordingly, we get the standardized results of each factor, and the score is linked with the spatial data to draw the spatial distribution layer of each factor (Table 4). All layers are converted to raster data with a resolution of $30 \mathrm{~m}$, the value of each pixel is the standard sore it gets.

Table 4. Standard scores of the factors

\begin{tabular}{cccccc}
\hline Standard scores & $\mathbf{1}$ & $\mathbf{2}$ & $\mathbf{3}$ & $\mathbf{4}$ & $\mathbf{5}$ \\
\hline I & $>50 \%$ & $35 \%-50 \%$ & $20 \%-35 \%$ & $5 \%-20 \%$ & $<5 \%$ \\
II & $\mathrm{N}$ & NE/NW & E/W & SE/SW & $\mathrm{S}$ \\
III & $>854 \mathrm{~m}$ & $640-854 \mathrm{~m}$ & $427-640 \mathrm{~m}$ & $213-427 \mathrm{~m}$ & $<213 \mathrm{~m}$ \\
IV & $<0.2$ & $0.2-0.4$ & $0.4-0.7$ & $0.7-0.9$ & $>0.9$ \\
V & - & Artificial & Semi-natural & Natural & - \\
VI & - & Coniferous & Broad-leaved & Mixed & - \\
VII & $\mathrm{T}_{1}$ & $\mathrm{~T}_{2}$ & $\mathrm{~T}_{3}$ & $\mathrm{~T}_{4}$ & $\mathrm{~T}_{5}$ \\
VIII & $>2000 \mathrm{~m}$ & $1500-2000 \mathrm{~m}$ & $1000-1500 \mathrm{~m}$ & $500-1000 \mathrm{~m}$ & $<500 \mathrm{~m}$ \\
\hline
\end{tabular}

Notes: 1, N, W, E and S represent north, west, east and south respectively, and they are applied with standard compass angle. $2, \mathrm{~T}_{1}$ includes brown coniferous soil, brown felt soil and stony soil. $3, \mathrm{~T}_{2}$ includes swamp soil and erosion type dark brown soil.4. $\mathrm{T} 3$ is thin layer of typical dark brown soil. $5, \mathrm{~T}_{4}$ includes medium and thick typical dark brown soil. $6, \mathrm{~T}_{5}$ includes meadow soil and river bank original dark brown soil 
Among them, the data of slope, aspect and elevation of Langxiang town can be collected directly or indirectly according to the DEM raster image provided by China Geological Cloud Data, and the forest-related data is from the Forest Distribution Map of Langxiang Forestry Bureau (2015). For soil type data, refer to Tieli Geological Survey Report (2016). Water distribution data is obtained by buffer calculation based on the Tieli City Land Use Status Database (2014).

\subsection{Spatial distribution of relative ES value}

By using the contribution rates and standard scores of each factor, we weight and superimpose the spatial distribution data of the factors with GIS, the spatial heterogeneity of ES indicators is calculated and visually expressed as raster images (Figure 4). Although the value of each pixel is a relative ES value range from 0 to 5 , it also clearly displays the spatial distribution of the ES function strength. In addition, since Chinese Forest Law clearly stipulates that all production activities cannot be carried out in special-use forests, these areas will be ignored in this study, same as the urban area, farmland, and water area.

The spatial distribution of ES value is relatively fragmented due to the multi-factor weighted overlay. However, by dividing the index scores by quantile thresholds, it can be found that the forest land with a higher value has a regular pattern of spatial aggregation, the reliability of the spatial results is confirmed through field investigation and survey.

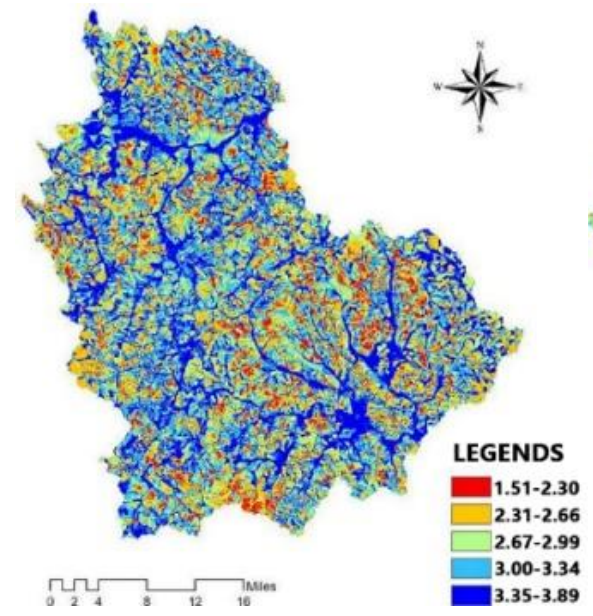

(a)

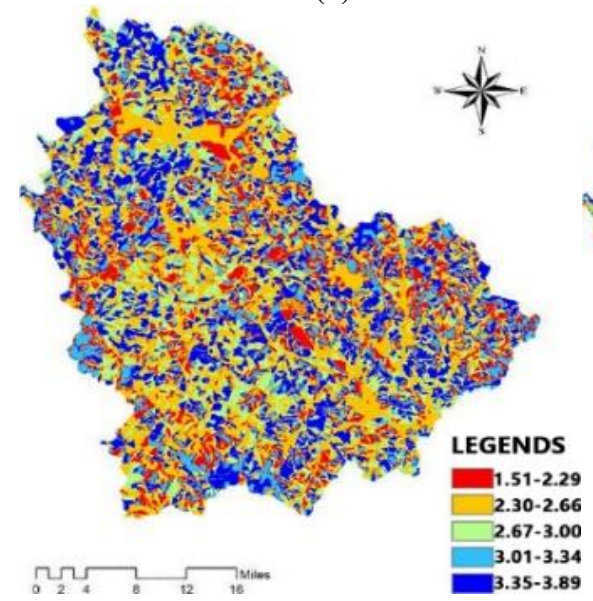

(c)

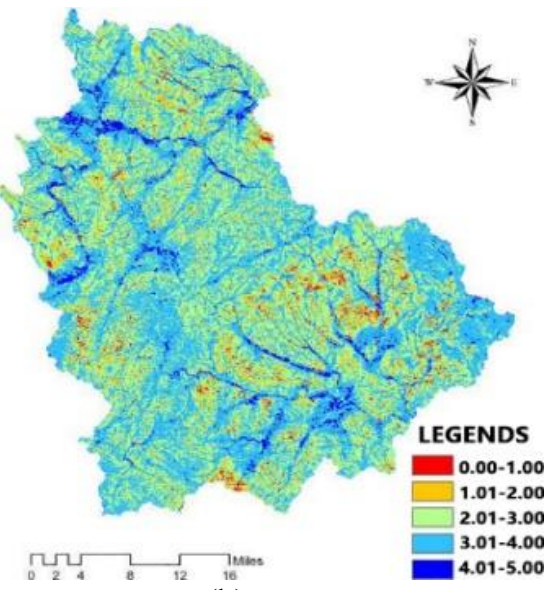

(b)

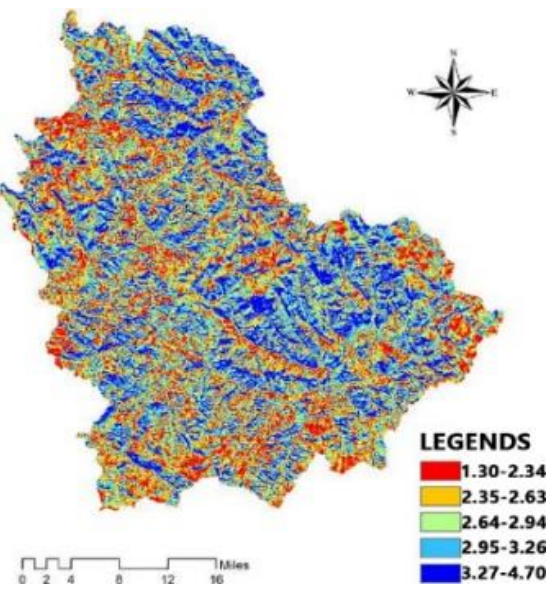

(d)

Figure 4. ES value spatial distribution map of indicator samples, (a) available forest land, (b) slope, (c) air purification and (d) winter landscape

\section{ES NEED DEGREE OF FUI}

According to the ES value map of indicators, the overall distribution of service level in the town can be judged as a whole. However, it cannot be used directly to conclude the development space for each type of FUI, because the relation of the ES functions is not simply additive. The supply level plays a key role in the industrial raw materials, the support level is a rigid condition index determining the setup of industries, and the regulation level is closely related to ecosystem security and the sustainability of industrial development. As a result of the different production conditions, production methods and product outputs of FUI, there are totally different needs for various ES functions. Moreover, the FUI is different from other ecological industries in that it has a high intensity of forest use, and has close energy and material exchange with the forest ecosystem, eventually the industries also become one part of the forest ecosystem. Therefore, for each type of FUI, a particular metric is necessary to fit the situation of ES, and we choose need degree to construct the metric. 
Referring to the current status and future development of the FUI in Langxiang, both with the Thirteenth Five-Year Plan for Forestry Development of the State Forestry Administration, we list 11 basic types of FUI. For each type, we comprehensive considered the space scale, favourable conditions, negative factors, and incidental pollutions of the production, combining with the experts survey, the need degree for related ES functions is calculated with a range of 0 to 5 , a greater value means a higher degree of need (Table 5). For the forest tourism, the supply service is replaced with the aesthetic landscape service when we calculate the needs.

Table 5. Need degree for ES indicators of different FUI types

\begin{tabular}{|c|c|c|c|c|c|c|c|c|c|c|c|c|c|c|}
\hline \multirow{2}{*}{ FUI types } & \multicolumn{3}{|c|}{ Supply } & \multicolumn{4}{|c|}{ Support } & \multicolumn{4}{|c|}{ Regulation } & \multicolumn{3}{|c|}{ Aesthetic landscape } \\
\hline & $\mathbf{A}$ & B & C & D & $\mathbf{E}$ & $\mathbf{F}$ & G & $\mathbf{H}$ & I & $\mathbf{J}$ & $\mathbf{K}$ & $\mathbf{L}$ & $\mathbf{M}$ & $\mathbf{N}$ \\
\hline Forest fungus cultivation & 2.5 & 4.0 & 1.3 & 2.1 & 3.2 & 2.4 & 3.9 & 3.6 & 0.7 & 4.4 & 4.6 & I & I & / \\
\hline Forest fruit planting & 3.7 & 4.1 & 3.9 & 2.8 & 4.3 & 2.9 & 4.3 & 4.5 & 0.6 & 3.8 & 4.6 & I & l & I \\
\hline Forest herbs planting & 2.6 & 4.0 & 0.8 & 1.3 & 3.9 & 3.6 & 4.8 & 4.6 & 0.7 & 0.5 & 4.9 & I & l & I \\
\hline Forest vegetable cultivation & 2.9 & 3.7 & 0.6 & 2.8 & 4.0 & 2.6 & 4.0 & 4.2 & 0.2 & 3.6 & 4.3 & I & l & I \\
\hline Forest poultry farming & 3.5 & 4.3 & 3.4 & 1.1 & 0.5 & 1.5 & 0.2 & 0.2 & 2.5 & 4.0 & 3.1 & I & l & I \\
\hline Forest livestock breeding & 4.1 & 4.5 & 3.6 & 2.0 & 0.7 & 2.0 & 0.2 & 0.2 & 4.0 & 4.0 & 3.1 & I & l & l \\
\hline Forest honey & 0.7 & 3.4 & 3.9 & 0.2 & 1.9 & 0.3 & 0.2 & 0.4 & 0.2 & 0.2 & 3.6 & I & l & I \\
\hline Nut harvesting & 0.2 & 4.8 & 1.2 & 0.9 & 0 & 0.8 & 0 & 0 & 0 & 0 & 0 & I & l & l \\
\hline Forest rafting & l & l & l & 4.3 & 0.6 & 4.0 & 0 & 0 & 0.5 & 1.5 & 2.4 & 5.0 & l & I \\
\hline Colorful Mountains & l & l & I & 1.3 & 1.6 & 1.0 & 0 & 0 & 0.8 & 1.5 & 2.0 & I & 5.0 & l \\
\hline Forest Skiing & l & l & l & 4.8 & 3.3 & 4.6 & 0 & 0 & 0.3 & 1.6 & 3.5 & I & / & 5.0 \\
\hline
\end{tabular}

\section{THE SPATIAL PATTERN OF FUI}

Convert the need degree into the weight values of the ES indicators, and the final weighted overlay is performed using equation (1) to obtain suitability zoning raster data of each FUI type. The value of each pixel is a suitability score, with a value range of 1-5. Take score ranged between 3-4 points as ordinary development area, more than 4 points as suitable development area, 2-3 points as restrictive area and other points as not suitable area, then we get the visualized partition maps. (Figure 5).

It can be seen from the maps that the quantity, spatial location, and dispersion degree of forest patches suitable for every industry are quite different, but they are generally concentrated within the scope of certain specific regions with the common features, which include the complex ES functions, centered on the valley line, rich biodiversity, and strong ecological stability and recovery capability.

The priority development area are selected and turned into vectorizations, we calculate them with Boolean operations to form a overall distribution map of all the FUI types, which is expressed as a superposition and combination with diffrent FUI patches. After a fine turning based on the economic scale benefits, location relationship, transportation accessibility, and construction of public service facilities, we clean up the tiny patches and adjust the boundary shapes. Thus, the spatial pattern of FUI development in Langxiang town is finally determined (Figure 6).

The spatial pattern result shows a guiding role in optimizing the industrial structure. We statistics the suitable area of all FUI types (Figure 7), and we find that some industries such as forest vegetable cultivation, nut harvesting and forest rafting, have greater suitable areas (more than $14.7 \%$ of the total area) than others significantly, and industries such as forest skiing and forest fungus cultivation, because of the strict production conditions or bigger destructiveness of forest ecosystem, the suitable areas are very limited (less than $4.3 \%$ ). In addition, the results also show that:

(1) Overall, high-quality forest resources suitable for developing FUI in Langxiang town are relatively concentrated, forming two agglomeration areas in the north and south, and the southern area are richer in types and larger in capacity.
(2) The spatial pattern of FUI is contrary to the urban space system based on timber harvesting. It can be seen that the industries which can be developed around the central town are scarce, and this area is only suitable for forest tourism activities such as forest rafting and forest skiing. However, the marginalized and slowly developed residential areas have greater potential for many types of FUI.

(3) Due to similar needs for ES functions, some suitable forestlands have a high degree of coincidence for multi types of FUI, these area can be identified as compound development zone, and it is applicable to new management concepts such as the forest farms with three-dimensional utilization. By this, the forest ES can be used more efficiently, besides that, the FUI can get more benefits through aggregation and collaboration.

(4) According to the spatial relationship between the major transportation routes, geographical divisions and industrial patches, we conclude five industrial axes, two forest tourism landscape belts and one forest skiing axe, this is the basic framework for future development of FUI.

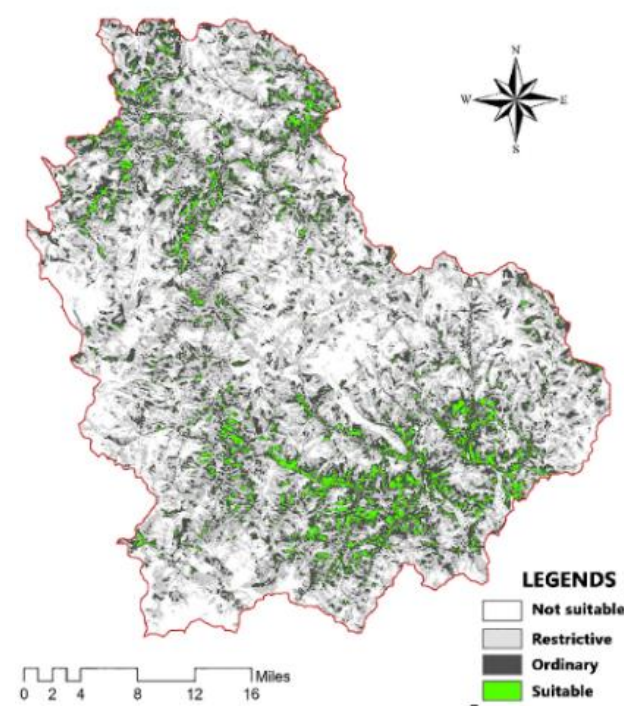

(a) Forest vegetable cultivation 


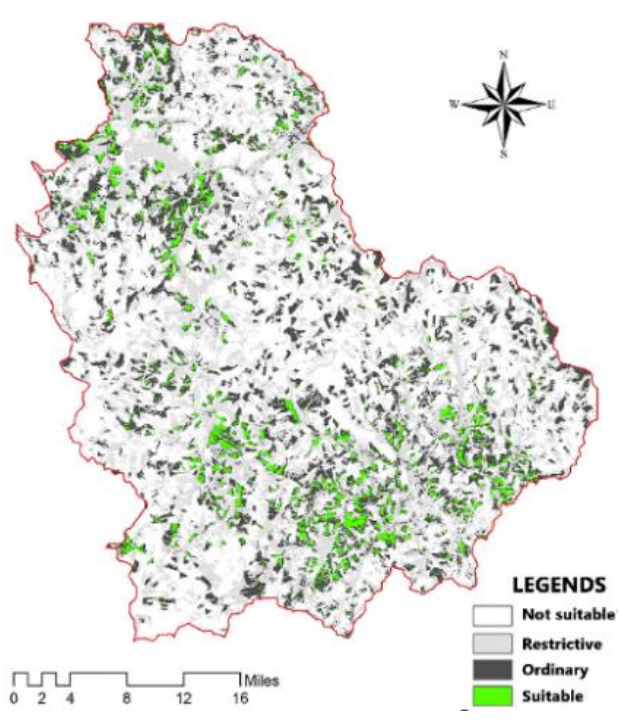

(b) Forest honey

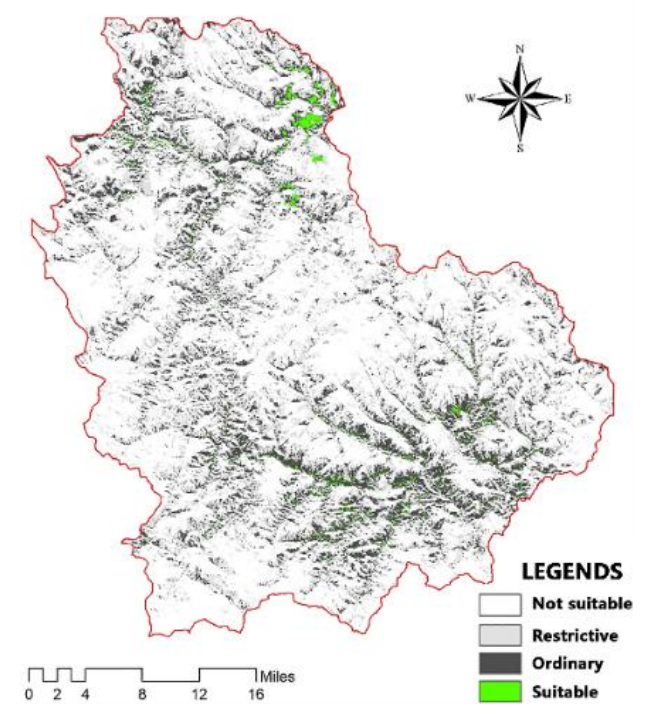

(c) Forest skiing

Figure 5. Samples of suitability zoning maps

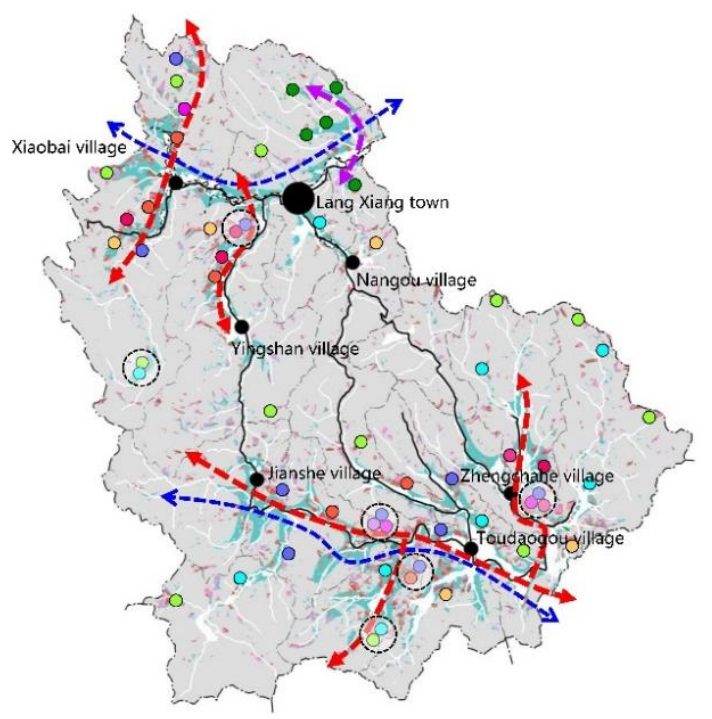

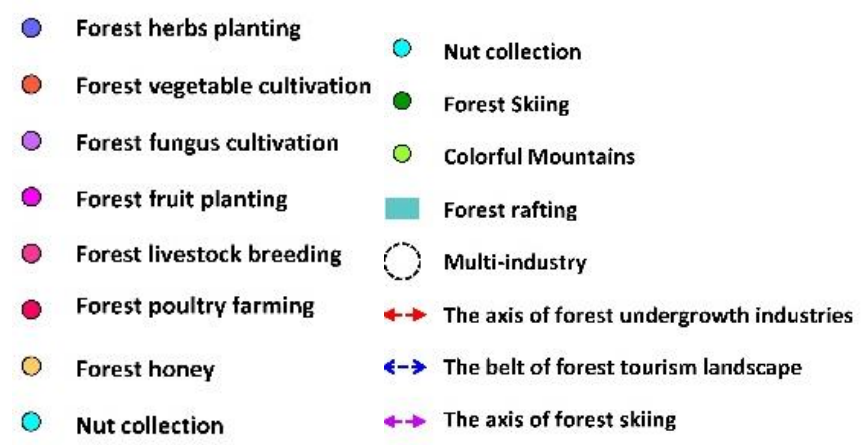

Figure 6. The spatial pattern of FUI based on ES value

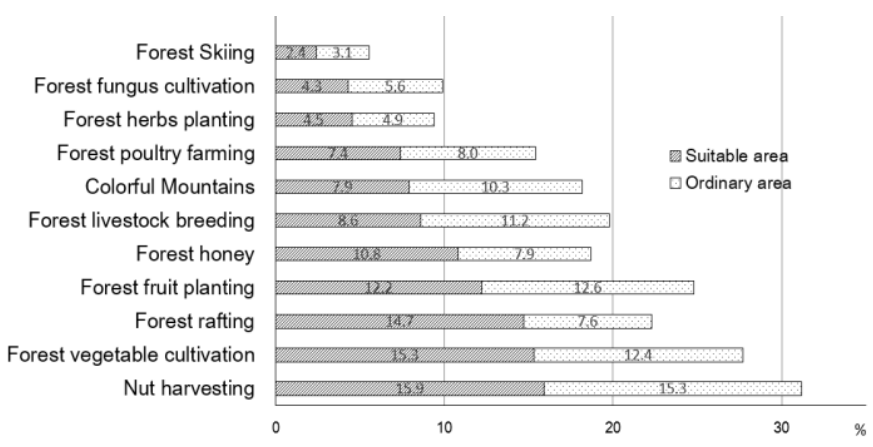

Figure 7. Percentage of FUI suitable and ordinary area

\section{CONCLUSIONS}

The development of FUI is the consequence of strict national forest ecological protection. In Langxiang, however, there is no effective measure or guidelines on the basic management of FUI, this led to a state of spread growth. A lot of FUI enterprises considered for the traffic and land costs, concentrated in the suburbs, while others only sought for the maximization of forest outputs ignoring the impacts on the ecological environment. The results of our study showed a more organic and reasonable industrial spatial structure, and it presents a very different state from the free development way.

Our research built up a process to combine the ES spatial heterogeneity with the environment condition requirement of FUI, the core goal of this is to excavate the possibility of guiding the spatial development with ES methods. The results showed a feasible way to organize the industrial area from chaotic to ordered and sustainable.

In our evaluation process, fewer data was used to describe the forest ES value because we mainly collected the spatial distribution information which is enough to achieve the goal of spatial pattern construction, and this method provide a convenient way for forest management. However, this method has a limitation in confirming the industry scale as a result of lack in accurate measurement of ES value. In future studies, we will try to solve not only the problem of "where", but also the problem of "how many".

\section{ACKNOWLEDGMENT}

This work is supported by the National key R \& D project "Research on low carbon planning and design of county towns based on regional characteristics and heritage" (2018YFC 0704705). 


\section{REFERENCES}

[1] Yu, D., Zhou, L., Zhou, W.M., Ding, H., Wang, Q.W., Wang, Y., Wu, X.Q., Dai, L.M. (2011). Forest management in northeast China: History, problems, and challenges. Environmental Management, 48: 1122-1135. http://dx.doi.org/10.1007/s00267-011-9633-4

[2] Gao, Y. (2015). Avoiding "resource curse" and constructing a new urbanization path under the new energy normalization, Taking the energy-rich area of northern Shaanxi as an example. Urban Planning, 39(10): 52-59.

[3] Fang, Z.F., Feng, B.C., Xu, G.F. (2010). Discussion on Developing Forest Undergrowth Industry: A Case Study in Thousand-isle Lake District. China Forestry Economy, 2010-04.

[4] Gao, H., Zhu, F., Li, R. (2008). Production Status and Development Trend of Walnut Processing Industry in China. Nonwood Forest Research, 2008-03.

[5] Costanza, R., d'Arge, R., de Groot, R., Farber, S., Grasso, M., Hannon, B., Limburg, K., Naeem, S., O'Neill, R.V., Paruelo, J., Raskin, R.G., Sutton, P., van den Belt, M. (1997). The value of the world's ecosystem services and natural capital. Nature, 387: 253-260. http://dx.doi.org/10.1038/387253a0

[6] Millennium Ecosystem Assessment. (2005). Ecosystems and Human Wellbeing: Biodiversity Synthesis. Washington DC: World Resources Institute

[7] Dobbs, C., Escobedo, F.J., Zipperer, W.C. (2011). A framework for developing urban forest ecosystem services and goods indicators. Landscape and Urban Planning, 99(3): https://doi.org/10.1016/j.landurbplan.2010.11.004.

[8] Zhao, T.Q., Ouyang, Z.Y., Zheng, H., Wang, X.K., Miao, H. (2004). Forest ecosystem services and their valuation in China. Journal of Natural Resources, 19(4): 480-491.

[9] Jonsson, M., Bengtsson, J., Gamfeldt, L., Moen, J., Snall, T. (2019). Levels of forest ecosystem services depend on specific mixtures of commercial tree species. Nature Plants, 5: 141-147. https://doi.org/10.1038/s41477-0180346-Z

[10] Xie, G., Zhang, C., Zhen, L., Zhang, L. (2017). Dynamic changes in the value of China's ecosystem services. Ecosystem Services, 26: 146-154 http://dx.doi.org/10.1016/j.ecoser.2017.06.010

[11] Fei, L., Shuwen, Z., Jiuchun, Y., Liping, C., Haijuan, Y., Kun, B. (2018). Effects of land use change on ecosystem services value in West Jilin since the reform and opening of China. Ecosystem Services, 31: 12-20. http://dx.doi.org/10.1016/j.ecoser.2018.03.009

[12] Rao, Y., Zhou, M., Ou, G., Dai, D., Zhang, L., Zhang, Z., Yang, C. (2018). Integrating ecosystem services value for sustainable land-use management in semi-arid region. Journal of Cleaner Production, 186: 662-672. http://dx.doi.org/10.1016/j.jclepro.2018.03.119

[13] Schmidt, S., Manceur, A.M., Seppelt, R. (2016). Uncertainty of monetary valued ecosystem services value transfer functions for global mapping. PLOS ONE, 11(3): e0148524. http://dx.doi.org/10.1371/journal.pone. 0148524

[14] Hu, X., Hong, W., Qiu, R., Hong, T., Chen, C., Wu, C., (2015). Geographic variations of ecosystem service intensity in Fuzhou City, China. Sci. Total Environ, 512513:

$215-226$. https://doi.org/10.1016/j.scitotenv.2015.01.035

[15] Wang, J., Zhou, W., Pickett, S.T., Yu, W., Li, W. (2019). A multiscale analysis of urbanization effects on ecosystem services supply in an urban megaregion. Science of the Total Environment, 662: 824-833. http://dx.doi.org/10.1016/ j.scitotenv.2019.01.260

[16] Li, M. (2014). Ph.D. dissertation. Ecosystem Services Evaluation Based on the InVEST Model: A Case Studies of Yanqing, Beijing. Beijing Forestry University, Beijing, China.

[17] Tieli city annals office. (2012). Tieli city, chapter 5, establishment. Xinhua publishing house.

[18] Zhang Manlin. (2016). Ph.D. dissertation. Research on land use change and its ecological environment effect in tieli city. Northeast Agricultural University, Harbin, China.

[19] Hui, G.Y., Wang, H.X. (2018). Reflections on the evaluation of forest ecosystem service functions. Temperate Forestry Research, 1(03): 5-9.

[20] Wu, X.F., Chen, J.D. (1995). The technical specification of forest resources planning and design investigation (category ii). Sichuan Forest Exploration and Design, 02: 37-41.

[21] Zhang, Y., Pan, J. (2015). A review of forest ecosystem service value assessment research. Forestry Economics, 37(10): 101-106.

\section{NOMENCLATURE}

ES Ecosystem services

FUI Forest undergrowth industries

ha $\quad 10,000 \mathrm{~m}^{2}$ 